\title{
The Material Traces Determination and Significance of Reprinted Belt Conveyor
}

\author{
Gui-Yu LIN ${ }^{1, a^{*}}$, Zhao-Yong LI ${ }^{2, b}$, Yu ZHOU ${ }^{3, c}$, Chao ZHANG ${ }^{1, d}$ \\ ${ }^{1}$ Liaoning Shenyang 11004 Mechanical Engineering and Automation of Northeastern University \\ China \\ ${ }^{2}$ Shandong Dongying 257000 The KERUI Group China \\ ${ }^{3}$ Liaoning Shenyang 110042 Engineering Design Institute of Northern heavy industries group China \\ alin_guiyu@163.com, blizhaoyong1990@126.com, czynhisk@163.com, d344410320@qq.com \\ ${ }^{*}$ Corresponding author
}

Keywords: Material Trace, Dynamics, Belt Conveyor, Impact.

\begin{abstract}
The determination of material traces is to meet the needs of the Subsequent production process and conveyor boom exciting force dynamics calculations. According to the conveyor's performance parameters, the thickness of the material layer can be known, in the course of calculating material traces; By the stress analysis of particles, the critical speed of conveyor belt to realize throwing motion model can be established. Respectively based on particle system dynamics theory and particle system momentum theorem to establish the center of mass velocity of the material flow, material flow trace mathematical models and material impact in the state of air resistance and no air resistance. To determine the basic parameters, a specific prototype is calculated based on the above principle. This provides a useful reference for follow-up design and work.
\end{abstract}

\section{The Significance of Material Trace Research}

The belt conveyer is very important in manufacture[1 2]. In the design of belt conveyor transfer device, the trace of materials and individual material blocks must be known when unloading the materials from the drum to ensure the feeder and the chute of reprinted device or keep-off column board and the installation position of buffer material conveyor idlers[3]; Determine the impact of the material reproduced to another conveyor to prepare for the boom conveyor dynamics calculation.

At present, there is no consideration or given ways to calculate the material layer method when determining the mathematical model of material traces. the material layer speed is replaced by the speed of the roller. This makes the results are different with the actual or lack of reliability.

The trace of bulk materials and reprinted conveying distance is related to the conveyor belt speed of material layer on the center of mass. And If the speed of the conveyor belt does not reach a certain value, the material does not have to be thrown out of the trend. The material's initial speed is the same with conveyor belt speed and it will fall vertically along the drum. The material will have the trend. To be thrown when the material is conveyed to the end of the drum when the speed of the conveyor belt $t$ reach a certain value, so the distance from conveyor to transfer conveyor can be determined.

\section{The Method of Determining the Bulk Material Traces}

The form of bulk material traces and reprinted conveying distance is related to the centroid velocity of material layer while the conveyor belt speed plays a decisive role.

Taking a particle from material layer for the study, analyze the stress and establish differential equation of motion by the function law of force. And then calculated according to the motion conditions of the problem to determine the motion of the particles.

\section{The Speed Model OF Material Break Away from the Conveyor Belt}

(1) The method of determining the thickness of the material layer 
Hypothesis: (1)There is no slip between the drive roller and the conveyor belt.

(2) The productivity of unit time as unchanged.

Approximate method for determining Material thickness $\Delta R$ : It's can be determined by productivity $Q$ $(\mathrm{kg} / \mathrm{s})$, belt speed $v$ (Or angular velocity $\omega$ ), bandwidth $B$, material density $\rho$, roller radius $R$, centriod velocity of the material layer $v_{\mathrm{c}}$.

After finishing, the material layer thickness is

$$
\Delta R=\frac{B \rho v R(-1 \pm \sqrt{1+4 k Q /(B \rho v R)})}{2 B \rho v}
$$

$\mathrm{k}$ : coefficient of material layer, $0<\mathrm{k} \leq 1 ; \mathrm{k}=0$, that, $\mathrm{s}$ no material layer, $\Delta R=0, \mathrm{v}_{\mathrm{c}}=\mathrm{v}$;

(2) The material from the conveyor belt speed model

That's the conveyor belt speed should reach a certain value, and this force was determined by the centrifugal force component in the vertical direction, the material can be thrown. The force of a particle is shown in figure 1.

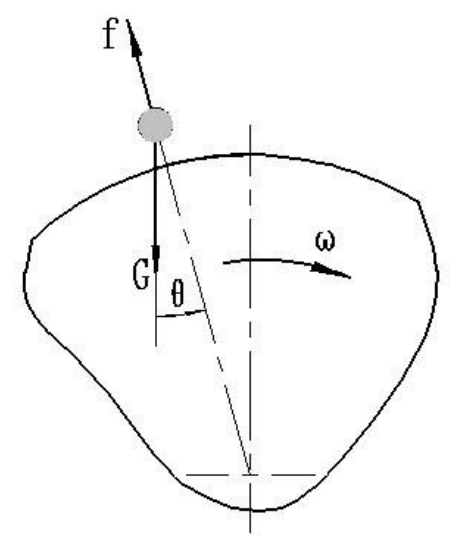

Fig. 1 Force Diagram of a Particle

The material on the drum is the particle system that is made up by material particles:

$$
f=m a_{n}=m g \cos \theta
$$

$v:$ conveyor belt speed, $\mathrm{m} / \mathrm{s} ; R$ : the roller radius, $m ; g:$ the acceleration of gravity, $g=9.8 \mathrm{~m} / \mathrm{s}^{2}$.

$$
\frac{v_{c}^{2}}{(R+k \cdot \Delta R) g} \geq \cos \theta
$$

Conveyor belt speed should meet the condition:

$$
v_{c} \geq \sqrt{(R+k \cdot \Delta R) g}
$$

Put the formula (1) into formula (4):

$$
v_{c}^{3}-R g v_{c}-\frac{k Q g}{B \rho}=0
$$

The critical speed that meet the type is

$$
v_{1} \geq \sqrt[3]{\frac{k Q g}{2 B \cdot \rho}+\sqrt{\left(\frac{-k Q g}{2 B \cdot \rho}\right)^{2}+\left(\frac{-R g}{3}\right)^{3}}}+\sqrt[3]{\frac{k Q g}{2 B \cdot \rho}-\sqrt{\left(\frac{-k Q g}{2 B \cdot \rho}\right)^{2}+\left(\frac{-R g}{3}\right)^{3}}}
$$

It's obvious that $\mathrm{v} \geq \mathrm{v} 1$, the material can be separated from the conveyor belt. 


\section{The Determination of Material Trace Model Having Air Resistance}

Thrown up the material with Inclined angle $\beta$ and initial velocity $\mathrm{vc}$, taking consideration of air resistance and no wind speed. Taking a particle from the material layer as the research object. Its coordinate system as shown in the diagram, and ensure the initial velocity of the particle in the plane oxy. As shown in figure 2.

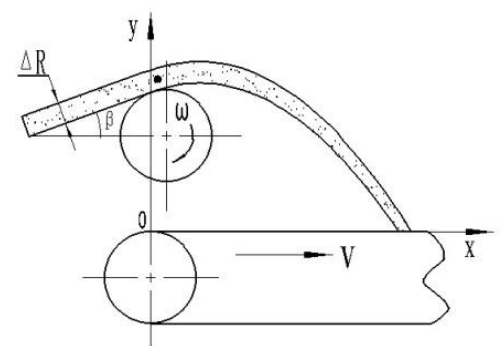

Fig. 2 The Material Trace Structure

$\beta$ : Angle of horizontal axis and the free fall starting material particle velocity direction; Initial condition:

$$
\begin{aligned}
& \text { 1) } t=0, v_{x}=v_{c} \cos \beta=(R+k \cdot \Delta R) \omega \cdot \cos \beta, v_{y}=v_{c} \sin \beta=(R+k \cdot \Delta R) \omega \cdot \sin \beta \\
& \text { 2) } t=0, x=0 ; y=H=(R+k \cdot \Delta R) \cos \beta+h ;
\end{aligned}
$$

In the above formula, $\omega$ : Angular velocity of roller, $1 / s, \omega=v / R$; $h$ : Distance of the roller center to the surface reprint on tape, $m$; $v$ : Speed of a layer of material, $\mathrm{m} / \mathrm{s}$.

Considering air resistance, its value is the material of the running speed of the center of mass of vc first power relations, namely air resistance $\mathrm{F}=\mathrm{c} \mathrm{mg} \mathrm{v}, \mathrm{c}$ for the known resistance coefficient; Is located in the trajectory of a particle for $\mathrm{m}$, the quality of its gravity $\mathrm{G}=\mathrm{mg}$; Its velocity direction and horizontal angle as the gamma.

For particle differential equations:

$$
\begin{aligned}
& m \frac{d^{2} x}{d t^{2}}=-F \cos \gamma=-c m g v_{c} \cos \gamma \\
& m \frac{d^{2} y}{d t^{2}}=-m g-F \sin \gamma=-m g-c m g v_{c} \sin \gamma
\end{aligned}
$$

Obtained from type (6):

$$
\frac{d \dot{x}}{\dot{x}}=-c g \cdot d t
$$

Obtained from type (7),

$$
\frac{d \dot{y}}{(1+c \dot{y})}=-g \cdot d t
$$

Based on the conclusion from type integral (9) (10),

$$
\begin{aligned}
& \dot{x}=C e^{-c g t} \\
& \dot{y}=\frac{D e^{-c g t}-1}{c}
\end{aligned}
$$

According to the initial conditions (1),

$$
\dot{x}=\frac{d x}{d t}=v_{c} \cos \beta
$$




$$
\begin{aligned}
& \dot{y}=\frac{d y}{d t}=v_{c} \sin \beta \\
& C=v_{c} \cos \beta \\
& D=c v_{c} \sin \beta+1 \\
& \dot{x}=\frac{d x}{d t}=C e^{-c g t}=v_{c} \cos \beta \cdot e^{-c g t} \\
& \dot{y}=\frac{d y}{d t}=\frac{D e^{-c g t}-1}{c}=\frac{\left(c v_{c} \sin \beta+1\right) e^{-c g t}-1}{c}
\end{aligned}
$$

Based on the conclusion from type integral (10) (11),

$$
\begin{aligned}
& x=-\frac{v_{c} \cos \beta \cdot e^{-c g t}}{c g}+C_{1} \\
& y=-\frac{1}{c g}\left(v_{c} \sin \beta+\frac{1}{c}\right) e^{-c g t}-\frac{t}{c}+D_{1}
\end{aligned}
$$

According to the initial conditions (2),

$$
\begin{aligned}
& x=\frac{v_{c} \cos \beta}{c g}\left(1-e^{-c g t}\right) \\
& D_{1}=H+\frac{1}{c g}\left(v_{c} \sin \beta+\frac{1}{c}\right) \\
& y=\frac{1}{c g}\left(v_{c} \sin \beta+\frac{1}{c}\right)\left(1-e^{-c c t}\right)+H-\frac{t}{c}
\end{aligned}
$$

The material trace model elimination time t, by type (12),

$$
t=-\frac{1}{c g} \ln \left(\frac{v_{c} \cos \beta-c g x}{v_{c} \cos \beta}\right)
$$

And plug in type (14), draw material trace model,

$$
y=H+\left(v_{c} \sin \beta+\frac{1}{c}\right) \frac{x}{v_{c} \cos \beta}+\frac{1}{c^{2} g} \ln \left(\frac{v_{c} \cos \beta-c g x}{v_{c} \cos \beta}\right)
$$

Material particles in the vertical plane of the release began to fall, when the material particle velocity for constant free fall, material throwing trajectory should be is a parabola.

Material layer in speed by type (10) (11) , and ask for any time of the material layer and speed.

$$
\begin{aligned}
& v_{h 1}=\sqrt{\left(v_{c} \cos \beta \cdot e^{-c g t}\right)^{2}+\left(\frac{\left(c v_{c} \sin \beta+1\right) e^{-c g t}-1}{c}\right)^{2}} \\
& v_{c}=[(R+k \cdot \Delta R) \omega]=v+k \frac{\Delta R}{R}
\end{aligned}
$$

Suppose type (8) for $y=0$, get,

$$
e^{-c g t}\left(v_{c} \sin \beta+\frac{1}{c}\right)+\mathrm{gt}-\left(H g c+v_{c} \sin \beta+\frac{1}{c}\right)=0
$$

Solution of the transcendental equation, fall to reproduce on the surface of the transport time $t$ can be obtained; using type (15) the speed at the end of the material can be obtained. 
Material throwing trajectory of horizontal distance

To (14) of $y=0$,

$$
H+\frac{c v_{c} \sin \beta+1}{c v_{c} \cos \beta} x+\frac{1}{c^{2} g} \ln \left(\frac{v_{c} \cos \beta-c g x}{v_{c} \cos \beta}\right)=0
$$

Solution of the transcendental equation, can get material casting trajectory of horizontal distance $\mathrm{x}$, in order to arrange feeder to feed slot position transfer device.

Horizontal distance to solve the material throwing tracks,

$$
x=\frac{v_{c}^{2} \cos \beta \sin \beta}{g\left(c v_{c} \sin \beta+1\right)}
$$

The type plug in (14), casting the maximum height of the material.

\section{Without Air Resistance Material Trace Model}

Without air resistance material trace model and with air resistance model with trace model, namely the air drag coefficient $\mathrm{c}=0$.

In the same way, it is concluded that the following parameters:

The displacement equation

$$
\begin{aligned}
& x=v_{c} \cos \beta \cdot t \\
& y=H+v_{c} \sin \beta \cdot t-\frac{g}{2} t^{2}
\end{aligned}
$$

Material down time : by type (20) for $\mathrm{y}=0$,

$$
t=\frac{2 v \sin \beta \pm \sqrt{(2 v \sin \beta)^{2}+4 g H}}{2 g}
$$

The maximal displacement $\mathrm{x}$ direction, $\mathrm{xmax}$, Suppose $\mathrm{y}=0$,

$$
x=\frac{\cos \beta \cdot\left(v_{c}^{2} \sin \beta+v_{c} \sqrt{v_{c}^{2} \sin ^{2} \beta+2 H g}\right)}{g}
$$

Resultant velocity

$$
\begin{aligned}
& v_{h 2}=\sqrt{v_{c}^{2}-2 v_{c} g \sin \beta \cdot t+(g t)^{2}} \\
& v_{c}=[(R+k \cdot \Delta R) \omega]=v+k \frac{\Delta R}{R}
\end{aligned}
$$

The trajectory equation for materials

$$
y=-\frac{1}{2} \frac{g \cdot x^{2}}{\left(v_{c} \cos \beta\right)^{2}}+x \tan \beta+H
$$

\section{The Impact of the Material}

Material layer is composed of $n$ particles, particle system applied impulse theorem and according to the collision of particle system recovery coefficient definition $\mathrm{e}=\mathrm{I} 2 / \mathrm{I} 1$, there is, $\mathrm{I} 2=\mathrm{e} \mathrm{I} 1$,

In the formula, e means coefficient of restitution, its value and transport belt tension, related to the size of the factors such as elastic modulus, $0<\mathrm{e} \leq 1$, intend to take $\mathrm{e}=0.1$; I1 refers to collision process begins the instantaneous impulse of collision, $\mathrm{I} 1=\sum \mathrm{m}$ vht. $\mathrm{I} 2$ means end of the collision process instantaneous impulse of collision. vh $t$, that is , at the end of the center of mass velocity of material layer. 
$I_{2}=e I_{1}=e \sum m v_{\mathrm{ht}}(\mathrm{KN})$

Because the material is continuously on the reproduced transport machine, can be considered a rectangular wave exciting force.

\section{Practical Calculation}

Known: a prototype process parameters: $Q=5616 \mathrm{t} / \mathrm{h}(1.560 \mathrm{t} / \mathrm{s})$, the bandwidth $\mathrm{B}=1.2 \mathrm{~m}, \rho=1.4 \mathrm{t} / \mathrm{m} 3$, roller radius $\mathrm{R}=0.5 \mathrm{~m}, \mathrm{c}=0.01$; Falling distance, $\mathrm{H}=1.5 \mathrm{~m}, \mathrm{v}=4 \mathrm{~m} / \mathrm{s}, \beta=20^{\circ}, \mathrm{e}=0.1, \mathrm{k}=1$.

\section{Calculation of Relevant Parameters}

Generation into the related formula will be known parameters, as the following table,

\begin{tabular}{|c|c|c|c|}
\hline \multicolumn{2}{|r|}{ Name } & Numerical & Fonmula \\
\hline \multicolumn{2}{|c|}{ Material layer thickness.m } & 0.173 & (1) \\
\hline \multicolumn{2}{|c|}{ Critical casting speed, $\mathrm{m} / \mathrm{s}$} & 2.87 & (6) \\
\hline \multirow{5}{*}{$\begin{array}{l}\text { With the ail } \\
\text { resistance }\end{array}$} & $\begin{array}{l}\text { The whereabouts of } \\
\text { time, } s\end{array}$ & 0.725 & (17) \\
\hline & Horizontal distance, $\mathrm{m}$ & 2.871 & $(18)$ \\
\hline & Maximum height ,m & 1.633 & $(18)(15)$ \\
\hline & $\begin{array}{l}\text { Speed at the end of the } \\
\text { material } \mathrm{m} / \mathrm{s}\end{array}$ & 6.632 & $(17)(16)$ \\
\hline & The impact . $\mathrm{KN}$ & 10.441 & $(26)$ \\
\hline \multirow{5}{*}{$\begin{array}{l}\text { Without the air } \\
\text { resistance }\end{array}$} & $\begin{array}{l}\text { The whereabouts of } \\
\text { time.s }\end{array}$ & 0.72 & (22) \\
\hline & Horizontal distance, $\mathrm{m}$ & 2.63 & (23) \\
\hline & Maximum height , m & 1.588 & $\begin{array}{l}(23) \\
(25)\end{array}$ \\
\hline & $\begin{array}{l}\text { Speed at the end of the } \\
\text { material , m/s }\end{array}$ & 6.875 & $\begin{array}{l}(22) \\
(24)\end{array}$ \\
\hline & The impact $\mathrm{KN}$ & 10.725 & $(26)$ \\
\hline
\end{tabular}

\section{Conclusion}

Through calculation and analysis above, the following conclusions:

(1) the air resistance of the velocity, displacement, material trace and influential impact value;

Greatly influenced by the increase of air resistance to impact and influence on the displacement is small;

(2) if the formation of casting speed, should make the smallest working speed of conveyor belt $\operatorname{vmin} \geq 2.87 \mathrm{~m} / \mathrm{s}$;

(3) the material flow casting line in a horizontal distance of $2.63 \mathrm{~m}$, the baffle end conveyor should be in the range arrangement;

(4)When there is no air resistance, the impact of the $10.725 \mathrm{KN}$, an exciting force is the rectangular wave type continuous function in the reproduced transport machine.

\section{Acknowledgements}

This work was financially supported by Chinese National 863 Project(2012AA062002).

\section{Reference}

[1]Zhao Yuwen, Li Yunhai, The present and future development in manufuture, The coal mine machine. 2004(4):1-3.

[2]Song Weigang, Wang Ying, The progress of the belt converyer, Hoisting and converying machinery, 2004(2):1-3.

[3]Ф.К. Yin wan, Qin Ke, etc, Shen Jingbao et al. Lifting transportation machinery computation. Beijing: China railway publishing house, 1982. P348 351. 